\title{
Automatic pelvis segmentation from x-ray images of a mouse model
}

\author{
Omar M. Al Okashi, Hongbo Du, Hisham Al-Assam* \\ Department of Applied Computing, University of Buckingham, \\ Buckingham, MK18 1EG, UK
}

\begin{abstract}
The automatic detection and quantification of skeletal structures has a variety of different applications for biological research. Accurate segmentation of the pelvis from X-ray images of mice in a high-throughput project such as the Mouse Genomes Project not only saves time and cost but also helps achieving an unbiased quantitative analysis within the phenotyping pipeline. This paper proposes an automatic solution for pelvis segmentation based on structural and orientation properties of the pelvis in X-ray images. The solution consists of three stages including pre-processing image to extract pelvis area, initial pelvis mask preparation and final pelvis segmentation. Experimental results on a set of 100 $\mathrm{X}$-ray images showed consistent performance of the algorithm. The automated solution overcomes the weaknesses of a manual annotation procedure where intra- and inter-observer variations cannot be avoided.
\end{abstract}

Keywords: Pelvis Segmentation, X-ray, Mouse Model image Segmentation

\section{INTRODUCTION}

The Mouse Genetics Project [1] was initiated to systematically knock-out mammalian genes and screen for a broad range of resulting traits. Over many years of research, mouse has been considered and accepted as a key model to assess mammalian gene functions because of its similarities to human ones in terms of genetics and physiology in addition to the feasibility of manipulating and analysing mouse genome [2]. One of essential element to monitor and assesses alterations in mouse model in the phenotyping pipeline is through using X-ray images. The skeleton of the mouse may involve important changes that may relate to diseases. Considering the number of images generated by the highthroughput screening process, an automated approach for annotating and triaging X-ray images will reduce processing time and costs on human resources, and eliminate possibilities of human error and inconsistency in manual measurement.

In the literature, the work dedicated to the mouse pelvis, especially in terms of automatic segmentation and annotation of mouse pelvis from X-ray images, if not at all non-existent, seems very limited. However, our previous works in [3] and [4] discussed the automatic phenotyping of different parts of mouse body in X-ray images. In [3] we presented an automatic method to segment and count the ribs of mouse from X-ray images to discover any abnormal counts resulting from gene knock-out. Another previous work in [4] proposed a fully automatic method to segment and quantify the curvature of the spine from the mouse X-ray images with aim to classify the scoliotic cases from normal one.

Some proposals to segment pelvis in different human medical images are existed. Xie et al. in [5] described framework based on SSM and statistical appearance model (SAM) for fully automatic pelvis segmentation from radiograph image. The method started by construction SSM and SAM from 100 manually annotated images. For a test image, the SSM was then registered coarsely with the pelvis and the SAM applied to select a candidate landmarks. Next, the deformation stage of the SSM was applied and repeated with the output landmarks from SAM until the algorithm converges. As those solutions could not achieve a satisfactory segmentation for some area in pelvis, they proposed using several anatomicalspecific information processing techniques such as adaptive threshold, median filtering, B-spline interpolation and geometric projection to enhance final segmentation results. Another framework to segment the pelvic bone from CT scan images was presented by Chandar and Satyasavithri in [6]. To enhance the image, the Gamma transformation was used to increase the contrast followed by median and smoothing filters to reduce the noises. Finally, a label filtering was used to select relevant contour and utilize them to segment the pelvic bone.

\footnotetext{
* hisham.al-assam@buckingham.ac.uk
} 
This paper proposes an automatic solution for pelvis segmentation based on structural and orientation properties of the mouse pelvis in the X-ray images. In this proposal, we interested in the segmentation of the two girdles bones of the pelvis. Experimental results on a data set of X-ray images confirmed the effectiveness of the proposed method with an overall accuracy up to $85 \%$.

\section{METHODOLOGY}

Our proposed solution consists of three main stages. The process starts with pre-processing the dorso-ventral 2D X-ray image that includes alignment, cropping of the pelvis ROI and then enhancing it. This is followed by segmenting an initial pelvis mask from the surrounding bones and other artefacts within the ROI. The method finally prepares an enhance pelvis image before masking it with initial pelvis mask to extract the final pelvis shape. The following subsections explain each step of solutions in more details.

\subsection{Pre-Processing}

The pre-processing step prepares the image for accurate pelvis segmentation. This step involves three operations: (a) aligning the mouse skeleton in a given image, (b) cropping the ROI, and (c) to enhancing the contrast of the cropped ROI. The inconsistent initial position of the mouse body in the input image as shown in Figure 1.a might complicate the later stages. The alignment operation is intended to bring the mouse body to a vertical position in order to enable accurate cropping of ROI. To perform the alignment, we adopt the method developed in our previous work in [4]. The method is based on locating two points on the mouse body after applying initial rotation (Figure 1.b) and then aligning the body by the angle that bring the line between the points $\mathrm{P}$ and $\mathrm{Q}$ to the vertical position as shown in the Figure 1.c. Further details of this method can be found in [4].

After aligning the mouse body to a vertical position, we followed the method developed in our previous work [3] to crop the pelvis ROI from the aligned image. The main steps of the method as follows. First, a limb-and-skull mask similar to that in Figure 1.d is obtained by applying the High Emphasis-Butterworth High Pass Filter followed by the region grow segmentation [7]. After that, four reference points, as highlighted by the blue points in Figure 1.e, were annotated by using the limb-and-skull-mask to estimate the ROI. Further details of this method can be found in [3].

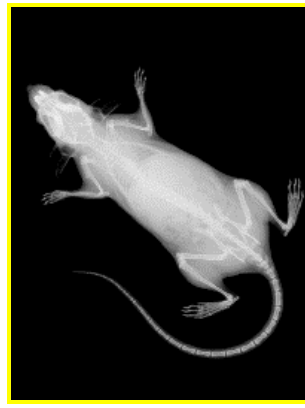

(a)

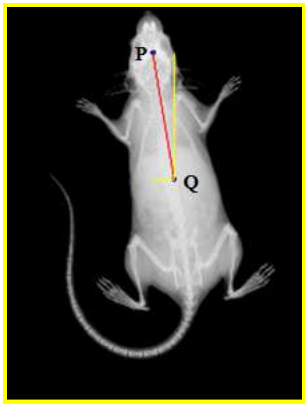

(b)

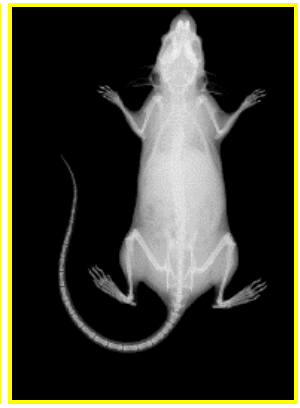

(c)

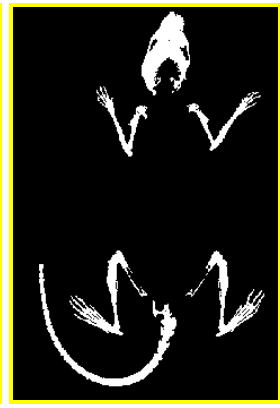

(d)

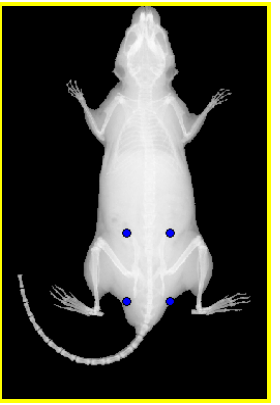

(e)

Figure 1 Alignment of mouse body.

(a) Input mouse X-ray image of full body dorso-ventral view before alignment, (b) P and Q key alignment points and the line between them annotated on the initially rotated mouse body, (c) Mouse body after rotating it by the obtained angle of rotation (d) limb-and-skull-mask and (e) four annotated landmarks to crop the pelvis ROI

As shown in Figure 2.a, the cropped ROI may have very poor contrast that may affect the accuracy of pelvis segmentation. To increase the contrast of the pelvis, we use the grey scale morphology operations [7] and the result as shown in Figure 2.b. 


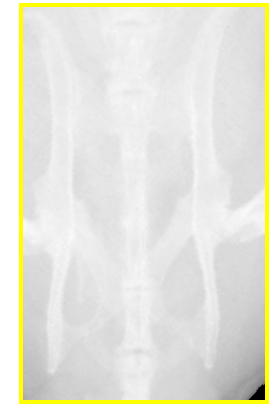

(a)

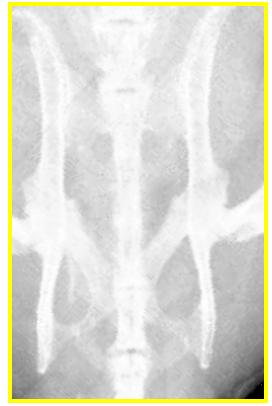

(b)

Figure. 2 Enhancing the contrast of the pelvis ROI.

(a) Initial cropped ROI before the enhancement, (b) enhanced ROI by using grey-scale morphology operations.

\subsection{Prepare pelvis mask}

This stage aims to prepare a binary mask for the pelvis in order to use it in the next stage to extract the pelvis. First, the Gabor filters with the set of angles between $\left(85^{\circ}\right)$ and $\left(95^{\circ}\right)$ is used to estimate the initial location of the pelvis girdles as shown in Figure 3.a. Gabor filters are bandpass filters which obtained by modulating a Gaussian envelope function with a sinusoidal wave and they are employed for feature extraction, edge detection and texture analysis in image processing [8]. The Gabor filter is chosen because in the aligned image the two girdles are most likely to be vertical. Next, the image in Figure 3.a is transformed to binary form using the threshold value generate by Otsu method [9] as shown in Figure 3.b. To denoise this image, non-pelvis objects are filtered out by comparing its features against some known feature values for the pelvis (i.e. Size, position and rotation angle) and. Finally, a closing binary morphology operations with $0^{\circ}$ and $90^{\circ}$ are applied to the binary image to build an initial pelvis mask shown in Figure 3.c.

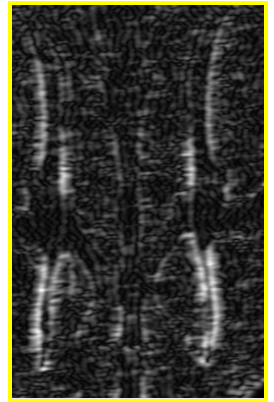

(a)

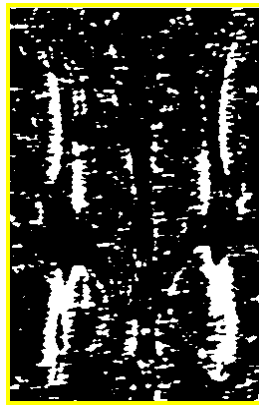

(b)

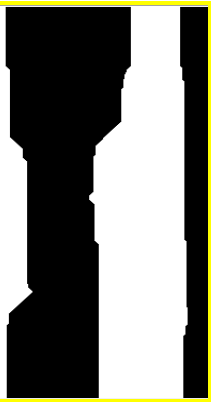

(c)

Figure 3. Initial pelvis mask preparation

(a) Result of applying Gabor filter; (b) Result of binary transformation; (c)Initial pelvis mask

\subsection{Pelvis segmentation}

The last stage of the pelvis segmentation starts with further processed the image in Figure 2.a with Gamma transformation [7] to increase its contrast as shown in Figure 4.a. Next, to exclude unwanted background noises around the pelvis, the grey scale morphology enhancement is applied on the image of Figure 4.a and the result is as shown in Figure 4.b. As it could be seen from Figure 4.b, the two girdles of the pelvis are very clear and distinctive from its background and all what is needed is to remove the pelvic vertebrae in the middle. To do so, a bit-wise multiplication is done between the initial mask of Figure 3.c and the enhanced version of the pelvis of Figure 4.b to produce the pelvis as shown in Figure 4.c. Finally, that segmented pelvis is transformed to binary mask using Otsu method. Figure 4.d shows the original image with pelvis girdles superimposed on top. 


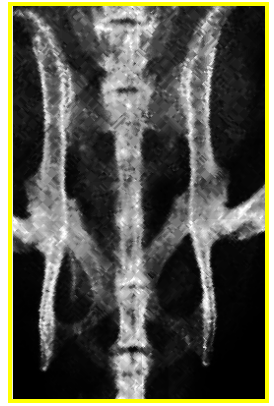

(a)

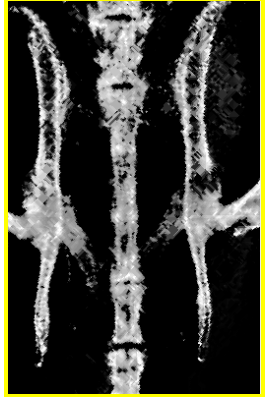

(b)

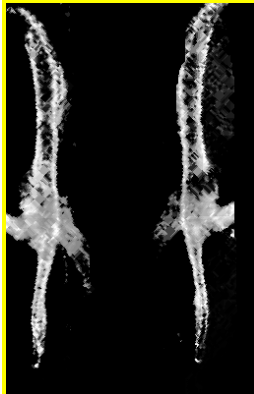

(c)

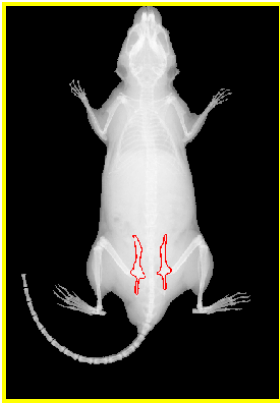

(d)

Figure 4. Final pelvis segmentation

(a) Result of applying Gamma transformation on enhanced contrast pelvis, (b) result of applying grey-scale morphology operations, (c)final segmented pelvis, (d) the segmented pelvis superimposed on the original aligned image

\section{RESULTS}

We have tested our method on a dataset of 100 full body dorsoventral view X-ray images. These 100 images include a variety of images of different degrees of difficulties in image analysis. For the purpose of this paper, our concern is to perform accurate segmentation of the pelvis girdle. To evaluate the segmentation of the results, we subjectively rated the quality of each image result as 'good', 'acceptable' or 'poor' by referring to several manually annotated images provided by an expert. Table 1 below shows the percentage of each level for each measurement.

Table 1. Accuracy of pelvis segmentation

\begin{tabular}{|c|c|c|c|}
\hline & Good & Acceptable & Poor \\
\hline Pelvis segmentation & $85 \%$ & $9 \%$ & $6 \%$ \\
\hline
\end{tabular}

The result is said to be 'good' if all or almost all of the pelvis girdles are segmented without extra noises as shown in Figure 5.a. In some cases, the whole required part of the pelvis is well segmented but with some small extra noise or some unwanted object segmented also. Such cases are rated as 'acceptable' quality as shown in Figure 5.b. The final 'poor' level happens when one or both girdles are missing either completely or partially as shown in Figure 5.c.

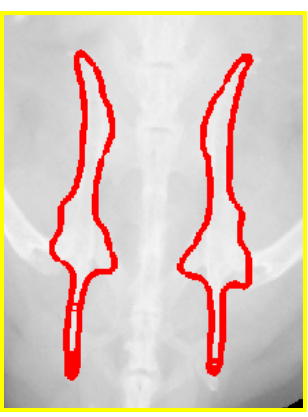

(a)

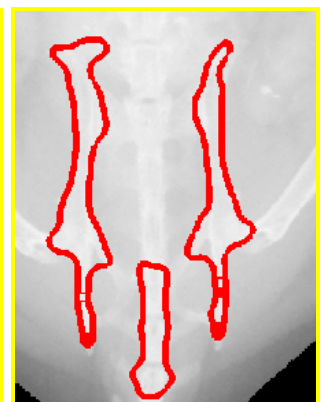

(b)

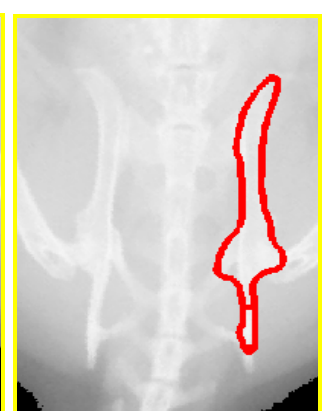

(c)

Figure 5 Accuracy of pelvis segmentation

(a) Good level pelvis segmentation, (b) Acceptable level pelvis segmentation, (c) Poor level pelvis segmentation

\section{DISSCUSION}

In general, all the proposed methods in this chapter showed good level of accuracy. Even with the fact that the quality check was done subjectively, the obtained results were considered as very encouraging, demonstrating the initial success of the proposed methods. Because of the bone segmentation from the X-ray images is not easy task, we can see that the accuracy of the pelvis segmentation is reasonable but not as high as we expected. One of the possible reasons that cause the 'poor' segmentation is the inaccurate cropping for pelvis ROI. One of the possible phenotyping that needed to measure from the pelvis is the flaring in the ischia, the base bone of the pelvis. Following the pelvis segmentation, this 
measure can be estimated automatically by calculating the distance between the lowest points of each pelvis girdle as shown in Figure 6. The figure shows the difference of the distance between unflared normal pelvis and flared one at the ischium pelvis.

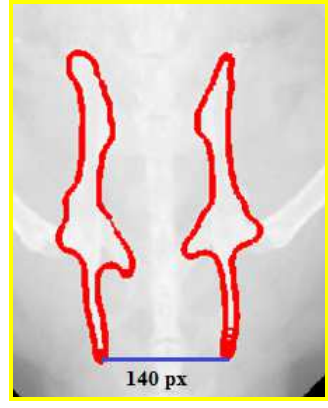

(a)

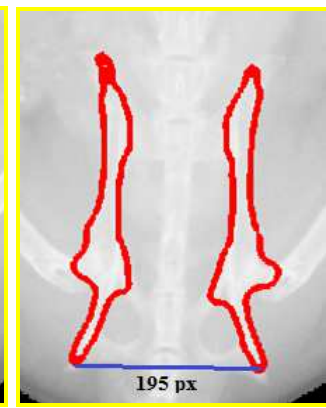

(b)

Figure 6 Measure the distance between the ischium of pelvis girdles

(a) The distance from unflared pelvis, and(b) the distance from flared pelvis

\section{CONCLUSION}

Accurate segmentation of the pelvis is important for assessing skeletal phenotype. We proposed a fully-automated algorithm that segments pelvis in X-ray images. An initial evaluation of the proposed methods on a limited collection 100 images indicates the promises of the proposed methods. The aim of this study was to locate the pelvis rather than extracting measures from it. We are now working to improve the level of accuracy of the segmentation. Our future work also includes a more in-depth analysis for the proposed schemes on a larger set of images. Once the proposed method is well tested and further finalised by using a much bigger data set, it can provide valuable information about specific phenotyping in the pelvis. Further investigation between the phenotyping results and genes will help to identify the genes responsible for the changes in pelvis shape between the mutant and wild type mice.

\section{REFERENCES}

[1] White, J.K., Gerdin, A.K., Karp, N.A., Ryder, E., Buljan, M., Bussell, J.N., Salisbury, J., Clare, S., Ingham, N.J., Podrini, C. and Houghton, R., "Genome-wide generation and systematic phenotyping of knockout mice reveals new roles for many genes," Cell, 154(2), pp.452-464 (2013)

[2] Spencer, G., "National Human Genome Research Institute (NHGRI)," 2012, <https://www.genome.gov/10005834/background-on-mouse-as-a-model-organism> (Accessed 27 July 2016).

[3] Al Okashi, O., Du, H., Selway, J.L., Lelliott, C., Maguire, S., Melvin, D., Langlands, K. and Al-Assam, H., “Automatic Ribs Segmentation and Counting From Mouse X-ray Images,". In MIUA ,143-148 (2014)

[4] Al Okashi, O., Du, H., Al-Assam, H., "Automatic Spine Curvature Estimation from X-ray Images of a Mouse Model," Computer Methods and Programs in Biomedicine, vol. 140, pp. 175-184, (2017).

[5] Xie, W., Franke, J., Chen, C., Grützner, P. A., Schumann, S., Nolte, L. P., Zheng, G., "A complete-pelvis segmentation framework for image-free total hip arthroplasty (THA): methodology and clinical study," The International Journal of Medical Robotics and Computer Assisted Surgery, vol. 11, no. 2, pp. 166-180, 2015.

[6] Chandar, K. P., Satyasavithri, T., "Segmentation and 3D Visualization of Pelvic Bone from CT scan Images," IEEE 6th International Conference, pp. 430-433, 27 Feberauary 2016.

[7] Gonzalez, R. C., Woods, R. E., [Digital Image Processing], 3rd ed., Pearson International Edition, 2008.

[8] Aach, T., Kaup, A., Master, R., "On texture analysis: Local energy transforms versus quadrature filters," Signal processing, vol. 45, no. 2, pp. 173-181, 1995.

[9] Otsu, N., A Threshold Selection Method from Gray-Level Histograms. IEEE Transactions on Systems, Man and Cybernetics, 9(1), pp. 62-66. 1979 